\title{
Digital Twin-Driven Framework for EV Batteries in Automobile Manufacturing
}

\author{
Rodrigo VALDEZ PARRA ${ }^{\mathrm{a}}$, Gaurav POTHUREDDY ${ }^{\mathrm{a}, 1}$, Tom SANITAS ${ }^{\mathrm{a}}$, \\ Vishnuvardan KRISHNAMOORTHYa , Oluwatobi OLUWAFEMI ${ }^{a}$, Sumit \\ SINGH $^{\mathrm{a}}$, Ip-Shing Fan ${ }^{\mathrm{a}}$, Essam SHEHAB ${ }^{\mathrm{b}}$ \\ ${ }^{a}$ Manufacturing Department, School of Aerospace, Transport \& Manufacturing, \\ Cranfield University, Cranfield, UK MK43 OAL \\ ${ }^{b}$ Mechanical and Aerospace Engineering Department, School of Engineering and \\ Digital Sciences, Nazarbayev University, Nur-Sultan, Kazakhstan
}

\begin{abstract}
The successful operation of Electric-Vehicle Batteries (EVB) is paramount for the ever-continuing goal of approaching a low carbon emission future. The Lithium-ion battery (LIB) is currently the best wager to implement on Electric Vehicles (EV). Nonetheless, it comes with its fair trade of challenges. The complexity involved in the design, manufacturing and operating conditions for these batteries has made their control and monitoring paramount. Digital Twin (DT) is concretely defined as a virtual replica of a physical object, process or system. The DT can be implemented in conjunction with the EVB physical embodiment to analyse and enhance its performance. ERP is a system designed to control production and planning amongst others. This paper presents the state-of-the-art battery design, production with the combination of DT and Enterprise system. A five-dimensional DT framework has been proposed linking the physical data and virtual data with ERP. The proposed method was used to model the digital twin of EVB at the concept level and solve its challenges faced in the industry Also the potential application \& benefits of the framework have been formalised with the help of a case study from Tesla EVBs.
\end{abstract}

Keywords. Electric-Vehicle Battery (EVB), Digital Twin (DT), Enterprise Resource Planning (ERP), Electric Vehicle (EV)

\section{Introduction}

In a bid to create a greener future driven by the net-zero commitment, manufacturing is presented with a significant role to play. Batteries costs contribute to almost half of the total cost of an electric vehicle [1]. EVB Manufacturers face the task of optimising the use of batteries, extending their life, improving safety and boosting the performance and efficiency, at a low cost. The next step in addressing the challenges identified is by using a collaborative utilisation of Digital Twin (DT) and Enterprise Resource Planning (ERP). A DT is essentially a virtual asset management system. It is created leveraging on machine learning, Artificial Intelligence, data modelling and simulation, using sensors, actuators ultimately for monitoring, and managing the total life cycle of an asset. On the other hand, an ERP is an integrated system that supports the easy flow of information from various

${ }^{1}$ Corresponding Author, Mail: g.pothureddy@cranfield.ac.uk. 
department in an organisation such as production, Material planning, human resources, and finance; it helps in the breaking down silos. ERP and DT together collectively provides the possibility of managing the EVB life cycle, supply chain and planning finance based on real accurate data and not forecasts. Existing research focuses majorly on optimising the EVB components particularly in a bid to reduce high costs however complex challenges in need of a wholistic approach persists. There is a gap in utilising the data to create value and enhance profitability in the EVB industry. This paper provides a digitaltwin framework that integrates ERP as a backbone for solving these challenges associated with the EVB.

\section{EV-B Landscape}

\subsection{Components of the Lithium-ion Battery Cell}

Batteries used in Electric Vehicles such as the famous automotive brand, Tesla, falls under the rechargeable Lithium battery class. The power generated from these batteries typically powers the functioning of the Electric vehicle, and they could be recharged at EV battery stations when they are low in power. The Lithium-ion battery cell consists of a positive electrode (cathode), a negative electrode (anode), a separator, and an electrolyte serving as a conductor. Carbonaceous materials such as artificial and natural graphite, mesophase and amorphous carbon and more recently silicon and tin oxides and alloys, as well as Lithium Titanium Oxide (LTO), are used as active anode materials [2].

The performance of the Electric Vehicle (EV) relies mostly on the performance of the EVB [3]. Some of the parameters identified to be vital in measuring the EVB performance include the charge capacity, energy density, cycle life, specific energy specific power as well as energy quality.

\subsection{Electric Vehicle Batteries (EVB)}

Due to its clean and sustainable system, EVs have started to get popular in demand. However, the essential element that forms the backbone for the functionality, reliability and profitability of the EV is the EVB, and there are a couple of challenges still faced in its manufacturing. To fulfil the high demand and expected SLA (Service Level Agreement) metrics for EVBs in the year 2030; lead times in the manufacturing of EVBs must be shortened while maintaining maximum quality and asset management. Amongst the numerous challenges faced by the EVB, this paper addresses five of them [4], as shown in figure 1 :

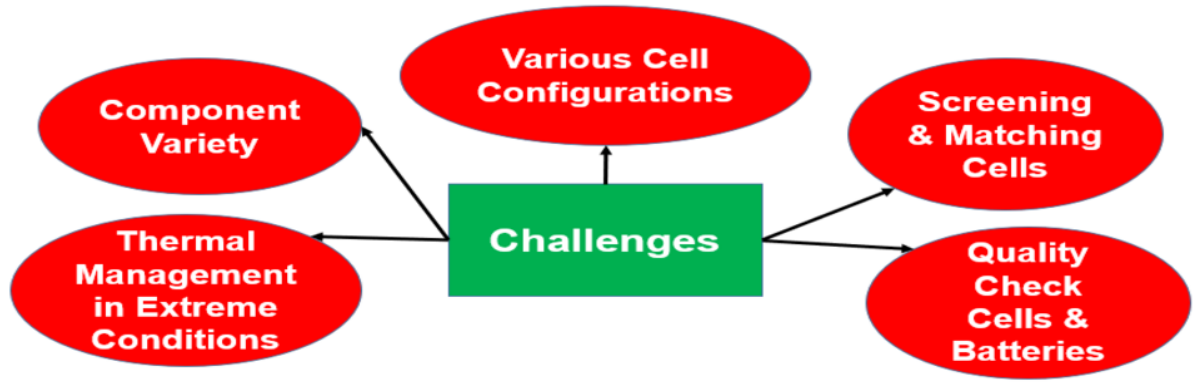

Figure 1. Challenges faced by EVB. 
Batteries are widely known to dissipate heat; the lithium-ion battery used in Electric Vehicles is no exception to this characteristic. Tesla, a well-known pacesetter in EV manufacturing, in a quest to optimise thermal management, has so far developed three generations of cooling/heating systems for its Vehicles. The first generation was a system that made use of glycol coolant [5] to pass through its cell module from one direction, serially, to another. The change in the second generation was that the glycol coolant had two entrances and two exits. Finally, the third generation involved the glycol coolant passing through the cell module in parallel. What's next? The fourth industrial revolution. Thermal management should become active as opposed to being passive. The thermal management challenge as well as the others are shown in figure 1, would be discussed further in the paper.

\section{Digital Twin}

\subsection{Concept of Digital Twin}

The concept of DT goes back to 2003 when Grieves [6] proposed the concept in his executive course on Product Lifecycle Management (PLM); DT was later introduced into the National Aeronautics and Space Administration (NASA) and the US Air Force. It was not until 2010 that an official concept for DT was brought up, in this case by NASA in their report "Draft Modeling, Simulation, Information Technology \& Processing Roadmap" [7]. DT is defined as "an integrated multiphysics, multiscale, probabilistic simulation of a vehicle or system that uses the best available physical models, sensor updates, fleet history, etc., to mirror the life of its flying twin" [7]. Grieves' initial concept focused on emphasising the continuous feedback and interactions between the virtual and physical entities. Three components are the core of his proposal: the already mentioned physical and virtual entities and the connection that allows the exchange of data between the two.

\subsection{Five-dimension Digital Twin}

Fei Tao's [8] proposed model builds on that of Grieve and expands on its definition and approach. Grieve's model keeps its focus solely on the physical-virtual interaction, Tao's enhanced proposal denotes a higher priority to the DT data which fuses the information acquired from both the physical and virtual entities for more capable information capture.

\subsection{Digital Twin in EVB Manufacturing}

DT has been defined many times, and to add one more to the dictionary, DT can be termed as "The mind of a machine", as shown in Figure 2. With an enormous and consistent flow of data, DT is enabling the manufacturing, operations and finance sector in a company to a higher limit. It not only tells how to manufacture an efficient product but also how to operate it. It covers the whole product lifecycle. DT represents the physical product in the digital world and performs tasks in the digital world, allowing us to do things faster while spending fewer resources, both monetary and workforce. For example, doing simulation instead of prototyping would be expensive and timely. DT 
can also predict the market analysis and help in cutting costs, grow operating margins and revenue. By making changes in the battery, it can give answers on the impact on margins, profitability, and return on investment. It can also improve a product's performance while reducing both the cost and risk of new product development [9].

\section{The Mind of a Machine}

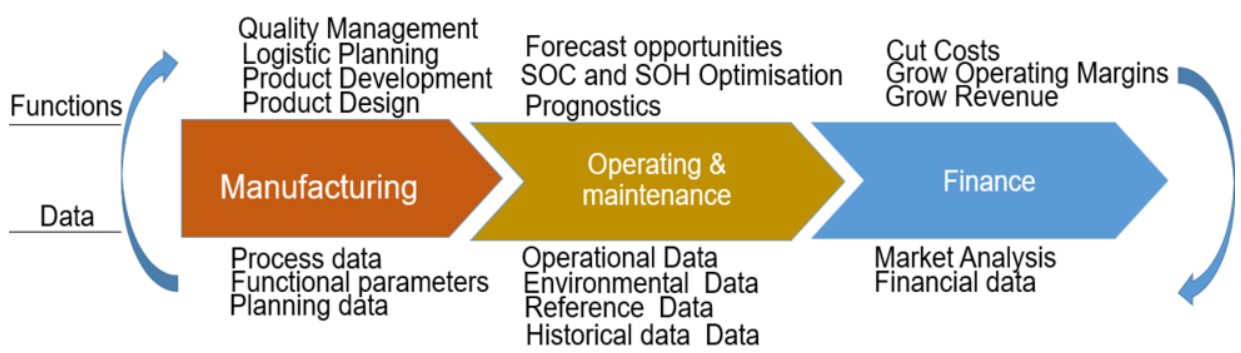

Figure 2. DT Mind of a Machine.

The DT will have its own architecture that through various simulation models would integrate engineering data, operational data and historical data. These models are going to be manually created and later modified by a model management system. Besides, the model management system can choose the best simulation model for relevant problems. [10]. It is also important to note that DT is also a feedback loop from operating to manufacturing, from operating into designing the next product generation, from the shop floor back into design, and so forth.

\subsection{Digital Twin Framework}

The framework for the proposal (Figure 3) is built on Fei Tao's five-dimensional DT [8][11], The Physical and Virtual data will be collected into the DT Data to be processed and analysed subsequently. The Connections between the components of the framework are enabled through the usage of dedicated ERP systems, which will lead to the services of the framework, churning out the optimisation and prognostics outcomes desired. Effective management of batteries can be approached by a basic State-of-X (SOX) estimation. The framework focuses on the basic key approaches of State-of-Charge (SOC) and State-of-Health (SOH) [12] of the batteries. These states determine the remaining useful lifetime (RUL) of a battery. This analysis incorporated with the other expected outcomes in services should enhance the work in addressing the established challenges.

The DT framework is conceptualised based on the following entities:

a. Physical Data: The data initially collected from the physical specimen is critical as it will set what can be gathered and inferred from the system through its analysis. The EVB will be monitored mainly for data on voltage, current, pressure, temperature and power consumption; environmental data is also recorded. 
b. Virtual Data: Gathered physical and previously recorded experimental data is utilised to create simulation models; built on models for geometry, physics, behaviour and rules. As their names state, the first two models label geometrical and physical properties, respectively. The behaviour models analyse the conduct of the product, user, environment and their interactions. The rules set the evaluation, forecasting and optimisations models [11].

c. DT Data: Data collected from the physical and virtual entities is stored in the 'DT Data'. This data will be further analysed and integrated; afterwards will be fed into the services and be used to execute the intended services of optimisation desired for the components.

d. Connections: All the components are bi-directionally connected through and between each other and associated by the ERP Systems. Facilitating communications through the usage of the established ERP Systems, ensuring all information communications are kept through a uniform system.

e. Services: Two types of services are considered, functional service (FS) and business service (BS) [13]. FS condenses the gathered DT data into the services that will verify the model assembly and verification, analytics, connection, communication and interaction [11].

The latter is managed to provide the desired outcomes found in the centre of Figure 3: SOC \& SOH Optimisation, Design, Manufacturing, Fault Diagnosis, Cell Balancing and Thermal Management.

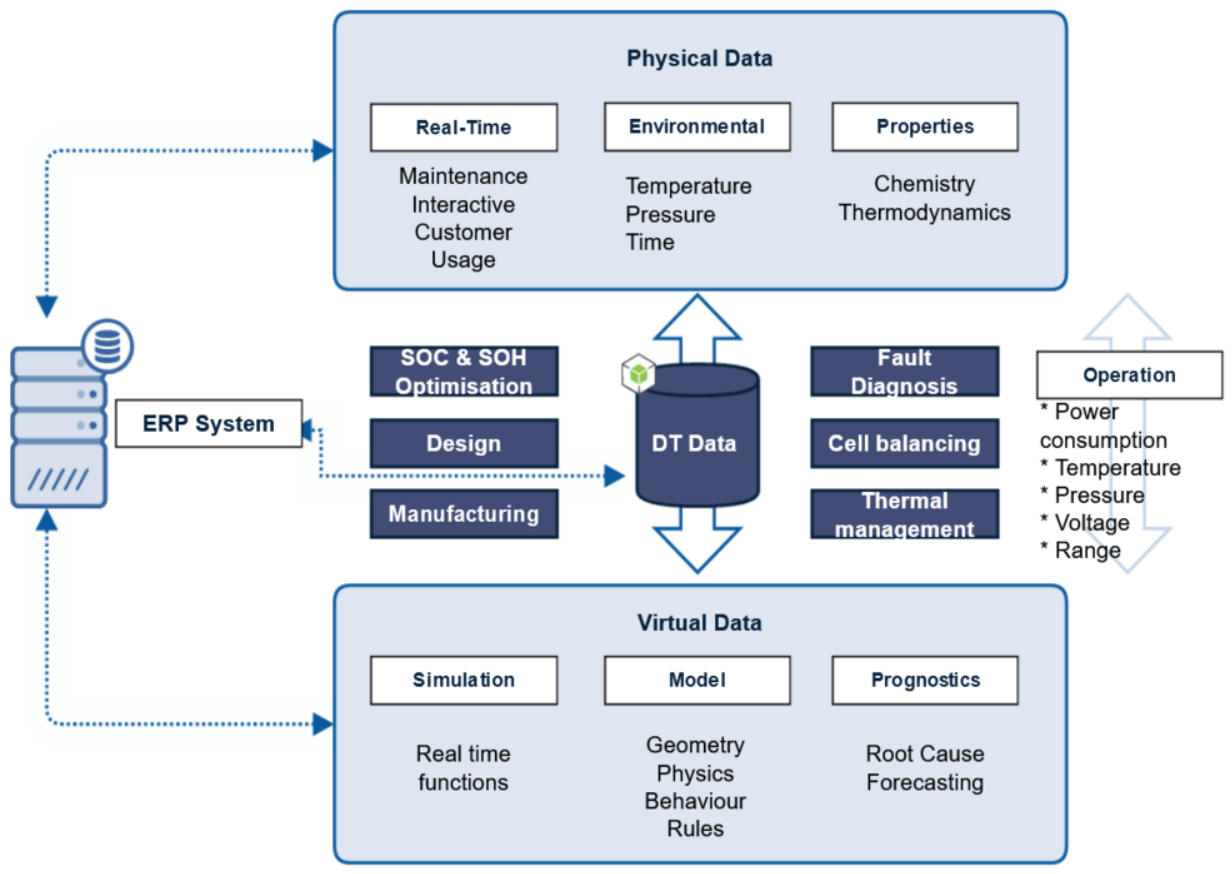

Figure 3. Digital Twin Framework. 


\section{Phase of ERP link to Digital Twin}

\subsection{ERP System}

Business administration ERP [14] systems that have an extensive collection of applications integrate and control all business activities of a corporation series features as shown in Figure 4 production, financial and accounting applications, Sales and distribution, project management, materials management, supply chain management (SCM), and quality management. The centralisation of information by the unified database is the central idea of the ERP method. This involves information from sales, marketing, development, and product design, field support, production, inventory control, management and procurement of industrial facilities, quality, logistics, human resources, finance, and database systems. ERP systems are software modules that share a centralised information basis and provide information.

- Production: ERP development module contains key features specially tailored for the engineering industry's unique needs. [15] It enables production preparation, orders, and delivers to consumers. It leads a company to manage personnel, equipment, and production scheduling optimally; it encourages the administrator to organise work as needed.

- Financial: The manufacturer will incorporate the financial reporting ERP method that can streamline payroll, outsourcing, production preparation, scheduling, and partnership. Through applying the ERP framework, the accuracy of financial data will enable quick decision-making [15].

- Inventory Management: ERP systems can manage the warehouse inventory level require. It helps define product needs, offers alternatives for replenishment, records inventory status, track material utilisation, establishes goals, and more. Material management module combines warning files with sales modules. Inventory enables procedures to manage a warehouse's acceptable stock volume.

- Sales: The ERP sales module is an essential module for the company. This ERP allows for the preparation, placement, transport, and invoicing of orders. It integrates with the website of the company, which makes communication more effective.

- Integration: Open Platform Communication Unified Architecture (OPC-UA) to connect the ERP and DT communication with the same production factors.

- $\quad$ Product Data Management (PDM): PDM provides product data management solutions for the duration of the product life cycle. Typically, PDM systems store CAD drawings and other designs ordered and well-documented archives.

- Product Lifecycle Management (PLM): PLM is a mechanism for handling through the stages of product life, from the development to the decline. The 
sectors of manufacturing and marketing are both handled. As the complexity of materials and supply chains increases, the manufacturing process becomes more difficult to handle. The main benefits of PLM are the increase the speed of getting the product to market, putting a higher quality, improve the safety. The PLM can be retrieved inside specialized computer software. The functions are documents managements, design integration and process management. [16]

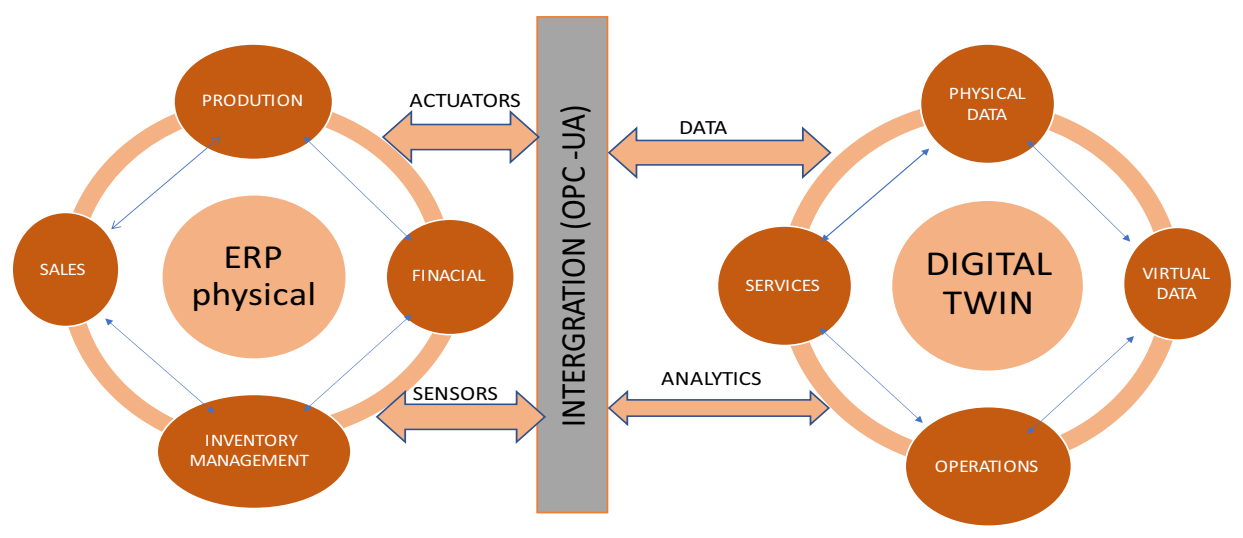

Figure 4. ERP \& DT link

\subsection{ERP and EV Challenges}

Previously, it has been explained that the integrated management software as ERP offers an interesting solution because they facilitate the management of the company's processes, and as a result, they increase organisational performance and individual performance. The main question revolves around the possibility of integrating an ERPtype solution to the challenges offered by Electrics Vehicle Batteries.

\subsection{ERP in the automotive industry}

The automotive manufacturer must respond to the new shifts that are facing them today. The data would play a vital role in the industry shift. It might not be surprising that in the future, the industry's supply chain and the production lines will likely tilt to the tech sector than to the manufacturing one as before [10]. The ERP could have an impact on the supplier by collecting data into a central hub, for example, lot tracking tools that could integrate data from digital assets and IoT devices. This system could be complemented by a PDM/PLM to treat the complex asset of data and drive the production development. But these systems will not be treated in detail in our study in order to focus on EVD-DT-ERP. 


\subsection{Answering EV Challenges}

Moreover, imagine that an ERP-type solution could help us solve the various challenges imposed by batteries. An ERP model will allow data to be centralised. These data will come from the different departments of the production line and would be easier to interpret. Designers should presume that considerable data is required for production design, voltage variance, and thermal management. Therefore, researchers may suggest a paradigm where data and perspective provided to us are at the core of the solution. This solution would be a System where a DT is implemented into an ERP-type model to answer EVB challenges. It can be assumed that DT could be implemented as software into the ERP, and this software would use the different data needed to do the experimentation and validation. The next step is the implementation of an EVB-DT-ERP solution.

\section{EVB-DT-ERP System}

Figure 5 shows how DT allows for blending data like customer feedback, design model, production data, engineering data, and maintenance data from enterprise applications such as ERP systems with the help of real-time data collected from the sensors placed in the physical battery. The DT can provide insights like SOC \& SOH of the battery, predictive analysis, strategy optimisation which will help in optimising the production chain of battery manufacturing and speeding up the decision-making process. This leads to increased profit margins because the design and manufacturing efficiency will proliferate when variables that could impede the process are identified and eliminated. The challenges mentioned in yellow boxes in Figure 5 are explained below and how DT can find a solution for the same.

1. The chemical reactions and cell physics that happens inside Lithium-ion batteries are quite complicated, and this is where DT can track the complex thermal behaviour and electrochemical changes. There are several viable positive electrodes each having distinctive characteristics. This variability presents the need for analysis for determining the best component use for functionality required. The analysis of historical data from similar cells and batteries makes it possible to predict the performance of a battery that has not yet been constructed. The DT allows you to use this information to model different scenarios by choosing the best pack assembly. i.e., the combination of series and parallel (which determines the charge capacity and power output) and finest battery components (active and inactive) for the new product and identify areas where new batteries can be improved over previous ones.

2. Variation of voltage in a cell is very critical and this can be avoided by creating a part twin of a cell, each part of the production process can be monitored and modelled by DT to identify where quality problems could occur and to evaluate the composition of the product being produced to determine whether there were any better materials and production processes that could have been used. By acting as a single point of reference for trusted information, DT can help resolve voltage variation issues between cells. 


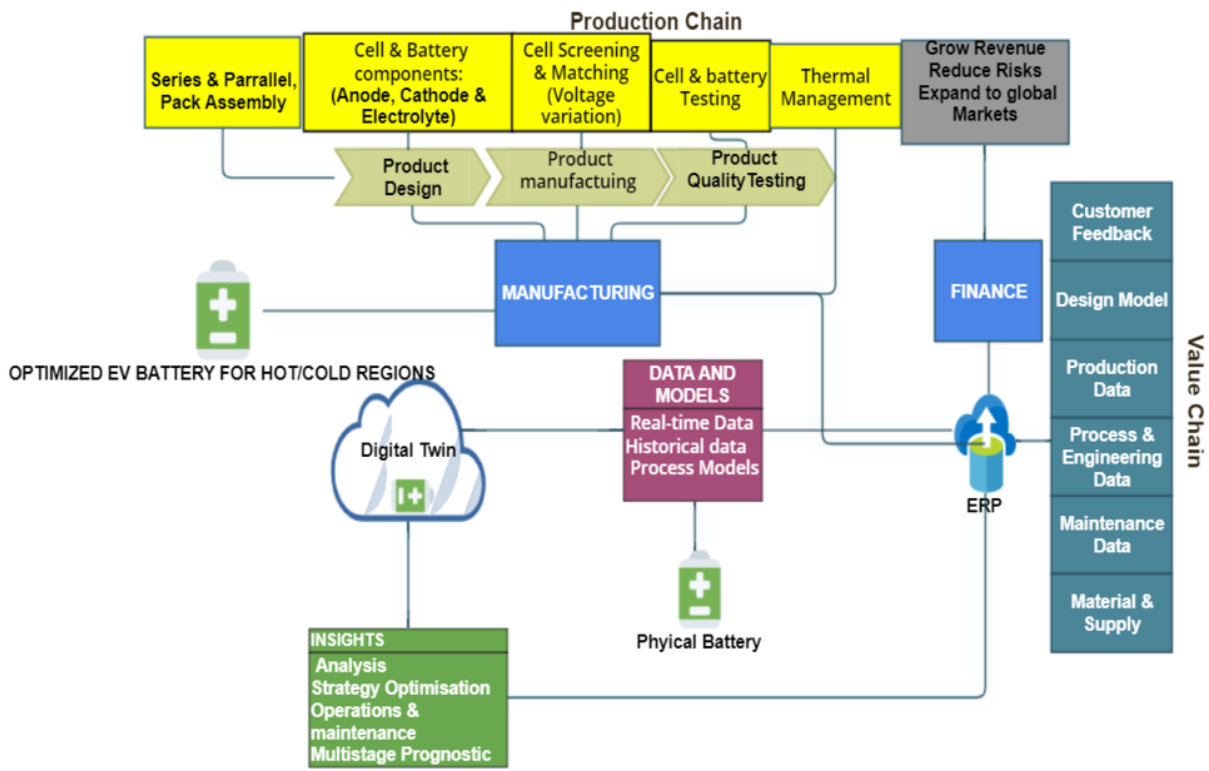

Figure 5. EVB-DT-ERP System

3. Thermal management is an exceptionally critical and significant challenge, Batteries are often optimised for either high or low temperatures and testing in relevant environment condition is not always possible. It can be computationally expensive and time-consuming, and this is where DT can perform various simulations in extreme conditions virtually and define the critical design parameters, key factors (heat generation and heat removal) and materials needed for optimal performance of the EV batteries in these conditions.

Hence by using our system companies like Tesla and EVB manufacturers who are facing challenges with the complexity of $\mathrm{Li}$ ion batteries can leverage upon this system to grow their business.

\section{Conclusions}

This paper aims to provide a reliable tool which can give investors more confidence and improve the business case, and in turn unlock more investments into EVB. This paper has addressed some of the key challenges faced by EVB. It proposes a framework of ERP integrated with digital twin for the monitoring of EVBs in hot and cold regions. Also, by Pairing real-world data with design simulation models it allows for good decisions based on realistic data on the other side. By leveraging on artificial intelligence, machine learning, and ERP, DT makes it possible to acquire insights and optimise the assets for maximum sustainability. This paper also presents a case study of Tesla, to show the current state of EVBs, their thermal management system and what benefits it could offer to improve them. Since all models and data are present in a coherent and well-aligned context, the concept of Digital Twin facilitates precisely such a process still 
needs further research to improve and enrich its capacity by considering more factors and practical situations.

\section{References}

[1] P. Wolfram and N. Lutsey, Electric vehicles: Literature review of technology costs and carbon emissions" Int. Counc. Clean ..., no. July, pp. 1-23, 2016, [Online]. Available: https://pdfs.semanticscholar.org/2913/dc7927c411ba6a922f426a67f01108699fd3.pdf\%0Ahttps://w ww.researchgate.net/profile/Nicholas_Lutsey/publication/305492926_Electric_vehicles_Literature review_of_technology_costs_and_carbon_emissions/links/5791a34c08ae6431.

[2] N. L. Franco, D. Persio, and L. Boon-Brett, Lithium ion battery value chain and related opportunities for Europe Title: Lithium ion battery value chain and related opportunities for Europe, 2014, [Online]. Available: ion_battery_value_chain_jrc105010.pdf.

[3] P. Roy, M. S. Akther Momin, and M.G. Kader, "Importance and Proper Way of Maintaining A Battery, International Conference on Mechanical Engineeringand Renewable Energy 2015 (ICMERE2015), 2015, November 2015, pp. 26-29.

[4] J. Jeevarajan, Separators used in Li-ion Cells Li-ion Cell Construction and Internal Protective Features, 2016, Acessed July 2021, https://thecityfixlearn.org/sites/default/files/UL\%20WRI\%20webinar\%20Li\%20ion\%20safety_Part $\% 202-\% 2816-30 \% 29$.pdf.

[5] G. Bower, Tesla Model 3 Battery Cooling Much-Improved ... Track Mode?, 2018. https://insideevs.com/news/338711/tesla-model-3-battery-cooling-much-improved-track-mode/ , accessed Dec. 10, 2020.

[6] F. Tao, M. Zhang, and A. Y. C. Nee, Digital Twin Driven Smart Manufacturing. Elsevier, Amsterdam, 2019.

[7] M. Shafto et al., DRAFT Modeling, Simulation, information Technology \& Processing Roadmap Technology Area 11, Natl. Aeronaut. Sp. Adm., p. 27, 2010, [Online]. Available: https://www.nasa.gov/pdf/501321main_TA11-MSITP-DRAFT-Nov2010-A1.pdf.

[8] F. Tao, M. Zhang, Y. Liu, and A. Y. C. Nee, Digital twin driven prognostics and health management for complex equipment, CIRP Ann., 2018, Vol. 67, No. 1, pp. 169-172.

[9] D. Gamota, How Digital Twins Lead to Manufacturing Profitability, https://www.jabil.com/blog/how-digital-twins-lead-to-manufacturing-profitability.html, accessed Dec. 06, 2020.

[10] M. Kunath and H. Winkler, Integrating the Digital Twin of the manufacturing system into a decision support system for improving the order management process, Procedia CIRP, 2018, Vol. 72, pp. 225-231.

[11] F. Tao, A. Liu, T. Hu, and A. Y. C. Nee, Digital Twin Driven Smart Design. Elsevier, 2020.

[12] B. Wu, W. D. Widanage, S. Yang, and X. Liu, Battery digital twins: Perspectives on the fusion of models, data and artificial intelligence for smart battery management systems, Energy AI, 2020, Vol. $1,100016$.

[13] F. Tao et al., Five-dimension digital twin model and its ten applications, Jisuanji Jicheng Zhizao Xitong/Computer Integr. Manuf. Syst. CIMS, 2019, Vol. 25, No. 1, pp. 1-18.

[14] A. Ullah, R. Bin, B. Khalil, M. Siddique, and A. Sami, Enterprise Resource Planning (ERP) Systems and User Performance (UP), J. Manag. Sci., 2017, Vol. XI, No. 03, pp. 377-390.

[15] H. Abualrejal, The Effectiveness of Enterprise Resource Planning- Erp Implementation In Manufacturing Industry, Proc. Symp. Technol. Manag. Logist. (STML-Go Green), 2016, Accessed: Dec. $09, \quad 2020 . \quad$ [Online]. Available: https://www.researchgate.net/publication/318445385_THE_EFFECTIVENESS_OF_ENTERPRIS E RESOURCE PLANNING-

[16] $\overline{\mathrm{T}}$. Segal, Product ${ }^{-}$Lifecycle Management (PLM) Definition, 2019. https://www.investopedia.com/terms/p/product-life-cycle-management.asp, accessed May 16, 2021. 